\title{
Étude internationale des curricula
}

Description d'un système complet de mesures du curriculum formel mis au point pour TIMSS

William H. Schmidt, Gilbert A. Valverde, Curtis C. McKnight et Edward D. Britton

Traducteur : Josette Le Coq

\section{OpenEdition} Journals

Édition électronique

URL : http://journals.openedition.org/ries/3436

DOI : $10.4000 /$ ries.3436

ISSN : 2261-4265

Éditeur

Centre international d'études pédagogiques

Édition imprimée

Date de publication : 1 décembre 1996

ISSN : 1254-4590

Référence électronique

William H. Schmidt, Gilbert A. Valverde, Curtis C. McKnight et Edward D. Britton, «Étude internationale des curricula ", Revue internationale d'éducation de Sèvres [En ligne], 12 | 1996, mis en ligne le 28 août 2013, consulté le 02 mai 2019. URL : http://journals.openedition.org/ries/3436 ; DOI : 10.4000/ ries.3436

(c) Tous droits réservés 


\title{
Étude internationale des curricula ${ }^{1}$
}

\section{Description d'un système complet de mesures du curriculum formel mis au point pour TIMSS}

\author{
William H. Schmidt, Gilbert A. Valverde \\ Curtis C. McKnight, Edward D. Britton ${ }^{2}$
}

La division du curriculum en trois composantes - curriculum formel (intended), mis en œuvre (implemented) et atteint (attained) - est depuis longtemps une caractéristique des études menées par l'IEA, (International Association for the Evaluation of Educational Achievement). Ce modèle à trois niveaux représente le curriculum comme une série de trois contextes emboîtés incluant des facteurs politiques et sociétaux, des activités concrètes pratiquées dans les classes et des résultats de l'enseignement. L'enquête sur les occasions d'apprentissage en mathématiques et en sciences (SMSO ${ }^{3}$ : Survey of Mathematics and Science Opportunities) a recueilli un ensemble de données qui traduisent de façon complète les trois aspects du curriculum pour la troisième enquête sur les mathématiques et les sciences (TIMSS ${ }^{4}$ : Third International Mathematics and Science Study). L'ampleur de l'étude et les technologies de

1 Cet article a été traduit par Josette Le Coq.

2 Les idées présentées dans ce papier reflètent quatre années de travail de l'équipe internationale de recherche de SMSO (the Survey of Science and Mathematics Opportunities). Les auteurs souhaitent remercier les autres membres de l'équipe pour leur contribution : É. Barrier (France), I. Gonzalo (Espagne), D. Jorde (Norvège), U. Moser (Suisse), K. Shimizu et T. Sawada (Japon), L. Burstein, S. Raizen, D. Wiley, R. Wolfe, L. Cogan et R. Prawat (USA). 3 L'enquête sur les occasions d'apprentissage en mathématiques et en sciences (SMSO) est menée par une équipe de recherche internationale formée d'un sous-ensemble de pays participant à TIMSS (la France, le Japon, la Norvège, la Suisse et les États-Unis). Sa fonction première était de mettre au point une grille conceptuelle pour aider à la construction des instruments de collecte des données. Elle fut aussi chargée de la conception, pour TIMSS, des techniques d'études du curriculum formel et des pratiques pédagogiques des professeurs. Les activités de SMSO et ses résultats dans le domaine de la caractérisation des pratiques pédagogiques dans un dispositif international, sont décrits dans : W. H. Schmidt, D. Jorde, L. Cogan, E. Barrier, I. Gonzalo, U. Moser, K. Shimizu, T. Sawada, G. Valverde, R. Prawat, C. McKnight, S. Raizen, E. Britton, D. Wiley et R. Wolfe : Characterizing Pedagogical Flow: An Investigation of Mathematics and Science Teaching in Six Countries, Boston/Dordrecht/London, Kluwer Academic Press, 1996.

4 Étude, récemment achevée, sur l'enseignement des mathématiques et des sciences, conduite sous la direction de l'IEA (International Association for the Evaluation of Educational Achievement) dans cinquante pays environ, ayant impliqué plus de 12650 établissements, 25300 professeurs et 655000 élèves. Elle comprenait l'étude détaillée des curricula (offres de cours, manuels, programmes et instructions officiels), de l'enseignement (occasions d'apprentissage, pratiques pédagogiques, formation des enseignants et objectifs des enseignants concernant la couverture des contenus), et de la réussite des élèves. 
mesure employées représentent une innovation importante pour les études IEA et plus généralement pour la recherche en éducation à grande échelle. Cet article décrit quelques-unes de ces nouvelles techniques, celles utilisées pour étudier le curriculum formel ${ }^{5}$.

\section{L'importance des mesures des objectifs curriculaires}

TIMSS reconnaît l'importance des spécificités nationales ou régionales des objectifs d'apprentissage, ces objectifs sont supposés constituer le curriculum formel (intended curriculum). Ils sont souvent inscrits dans des documents officiels comme les programmes et instructions (curriculum guides). Cependant ces documents varient dans leur façon de préciser les objectifs d'apprentissage. Les responsables ministériels et autres spécialistes d'un pays sont aussi des sources d'information sur la façon dont on espère que les élèves apprennent. Malgré leur absence de caratère officiel dans beaucoup de cas, les manuels scolaires, en tant que guides pour les professeurs et les élèves dans la mise en œuvre des intentions officielles, fournissent des informations supplémentaires sur les objectifs d'apprentissage.

SMSO a mis au point une série de techniques de mesure, l'analyse du curriculum de TIMSS (TIMSS curriculum analysis), pour effectuer la première étude empirique du curriculum formel en mathématiques et en sciences, jamais utilisée à grande échelle.

Le curriculum formel, en spécifiant les objectifs d'apprentissage au niveau national ou régional, met l'accent sur certaines pratiques possibles d'apprentissage et en limite d'autres. Par exemple, dans un pays avec un curriculum national obligatoire relayé par les évaluations nationales et un inspectorat des établissements, l'inscription d'un objectif d'apprentissage dans ce curriculum ne garantit pas qu'il sera atteint - c'est-à-dire que l'occasion d'apprentissage sera réellement offerte en classe - mais cela doit considérablement augmenter la probabilité qu'il le soit.

De même, l'absence d'un objectif augmente la probabilité que les pratiques possibles d'apprentissage en liaison avec cet objectif ne soient pas mises en place, mais comme précédemment, nous ne traiterons que des occa-

5 Les résultats et l'analyse de la méthodologie sont publiés dans les premiers rapports internationaux de l'étude du curriculum de TIMSS : W. H. Schmidt, C. McKnight, G. A. Valverde, R. T Houang et D. E. Wiley, Many Visions, Many Aims: A Cross-National Investigation of Curricular Intentions in School Mathematics, Boston/Dordrecht/ London, Kluwer Academic Press, 1996 ; W. H. Schmidt, S. A. Raizen, E. D. Britton, L. J. Bianchi et R. G. Wolfe, Many Visions, Many Aims: A Cross-National Investigation of Curricular Intentions in School Science, Boston/ Dordrecht/London, Kluwer Academic Press, 1996. 
sions d'apprentissage en termes de probabilité - distribution de probabilités des pratiques d'apprentissage possibles - et non de la certitude que ces occasions ne soient pas offertes.

Les différences, d'un pays à l'autre, dans la description des objectifs d'apprentissage et les politiques liées à ceux-ci, sont très importantes pour la compréhension de ces relations. La description, au niveau du système éducatif, des objectifs d'apprentissage donne des paramètres grâce auxquels les pratiques d'apprentissage possibles sont cadrées, bien que peut-être à des degrés différents, quel que soit le type de système.

Les sources d'information utilisées par TIMSS pour mesurer le curriculum formel sont les programmes et instructions, les manuels et les spécialistes du curriculum. En utilisant ces sources, le projet d'analyse des curricula de TIMSS a mis au point un ensemble de procédures qui comprennent des analyses détaillées des programmes et manuels pour le niveau le plus haut des populations cibles de l'enquête ${ }^{6}$. Les procédures prévoient aussi le suivi de la couverture d'un thème (tracing topic coverage) tout au long de la scolarité. Des questionnaires aux spécialistes du curriculum font aussi partie de l'ensemble des données. Ces procédures sont toutes liées par un dispositif, appelé grilles d'analyse des curricula de TIMSS (TIMSS curriculum frameworks), définissant tous les éléments curriculaires.

TIMSS considère le curriculum formel comme un élément de base définissant les pratiques possibles d'apprentissage. Les particularités d'un curriculum sont étroitement liées à la politique éducative et aux objectifs de qualité de l'éducation. Leur caractérisation internationale à l'aide d'un ensemble détaillé de mesures permet de juger de l'étendue des visions et desseins nationaux qui existent dans le monde et fournit un élément fondamental pour la compréhension du contexte dans lequel les curricula sont mis en œuvre.

Le premier but de l'analyse curriculaire de TIMSS est de répondre à la question: qu'espère-t-on que les élèves apprennent en mathématiques et en sciences? L'étude des objectifs curriculaires nationaux ou régionaux fournit la base fondamentale grâce à laquelle on peut interpréter, pour un pays, les structures des occasions d'apprentissage des disciplines données.

6 TIMSS a mesuré la réussite en mathématiques et en sciences des élèves de trois populations. L'analyse des curricula de TIMSS porte son attention sur ces trois populations à cause du lien primordial avec les mesures de réussite. En particulier, les manuels furent analysés dans leur intégralité seulement pour les niveaux les plus élevés de ces trois populations. La population 1 est formée des deux niveaux scolaires contenant le plus d'élèves de 9 ans. La population 2 est formée des deux niveaux scolaires contenant le plus d'élèves de 13 ans. La population 3 est formée des élèves en dernière année de l'enseignement secondaire. Une sous-population importante de la population 3 est formée des élèves spécialistes de mathématiques, de physique ou des deux. 


\section{Les grilles d'analyse des curricula de TIMSS}

TIMSS comporte une grande collection de données venant des centres nationaux participant à cette étude, une analyse des documents curriculaires, une série de questionnaires et des tests complexes de connaissance. L'ampleur de l'effort qui fut fourni dans les pays participants et dans plusieurs sites internationaux demandaient que toutes les composantes de TIMSS soient liées par un cadre

\section{Figure 1. Catégories de contenu des grilles d'analyse de mathématiques et de sciences}

Chaque volet d'une grille d'analyse est divisé en un ensemble de catégories principales. Chaque catégorie principale contient une ou plusieurs sous-catégories de niveau plus précis. La figure montre les catégories principales de contenu avec quelques sous-catégories décrites en détail pour donner un meilleur aperçu de la structure des grilles.

Grille d'analyse des mathématiques

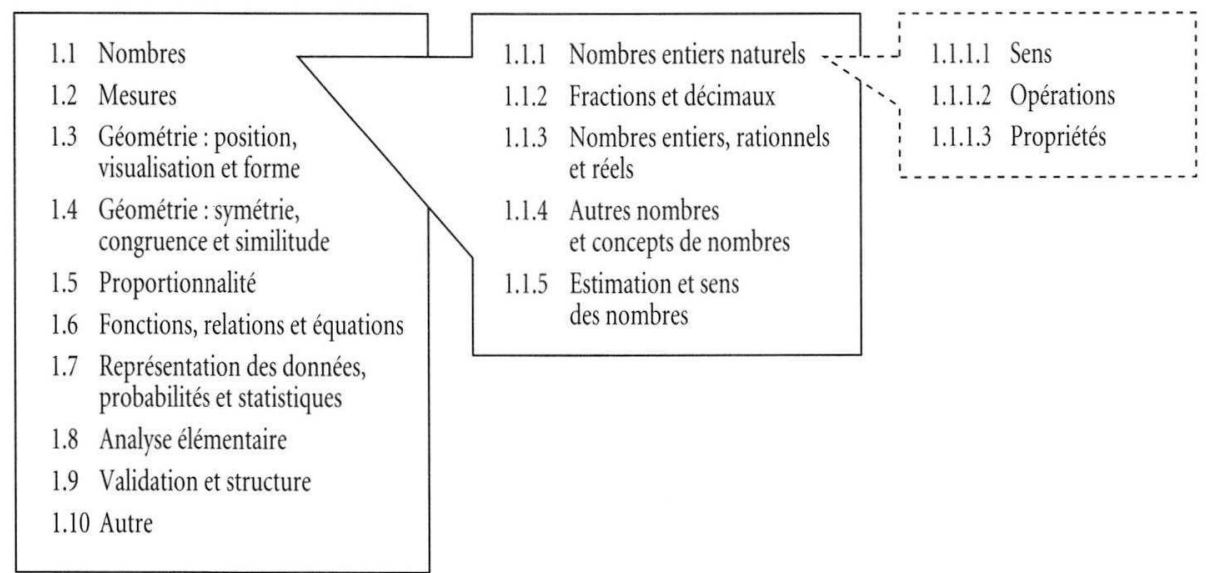

Grille d'analyse des sciences

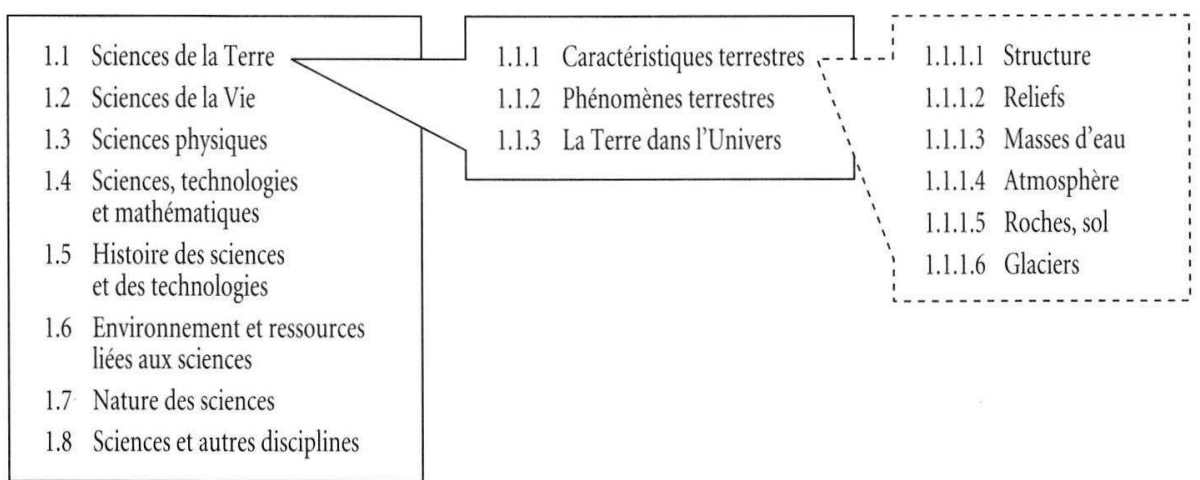


et un langage communs d'analyse. Que ce soit la classification d'un item des tests, la caractérisation d'une partie d'un document curriculaire ou la liaison entre un questionnaire et d'autres parties de TIMSS, toute description doit utiliser des termes et des catégories identiques, des procédures standardisées comme les codes numériques pouvant être recueillis dans des bases de données appropriées.

Deux grilles ${ }^{7}$ d'analyse - l'une pour les mathématiques et l'autre pour les sciences - fournissent le langage commun. Chacune d'elles couvre la scolarité complète dans un système unifié de catégories.

Chaque grille d'analyse a de multiples facettes et niveaux. Elle prend en compte les trois volets d'une discipline - contenus (thèmes disciplinaires), compétence espérée (ce que l'on espère que les élèves sachent faire sur un contenu particulier) et perspective (attitudes vis-à-vis d'une discipline et sa place parmi les autres disciplines et dans la vie de tous les jours).

Chaque grille d'analyse a été conçue pour utiliser des catégories multiples. En d'autres termes, tout élément - programmes et instructions nationaux, parties de manuels, items des tests - peut être classé dans autant de catégories de la grille d'analyse que nécessaire pour rendre compte de sa richesse. Chaque élément a une unique, parfois complexe, "signature " - un ensemble de contenus, compétences espérées et perspectives qui le caractérise selon les trois volets de la grille d'analyse. Ce système est souple, autorisant des signatures simples ou complexes selon le cas.

Cette multidimensionnalité flexible est essentielle pour analyser les documents curriculaires. Caractériser les curricula demande un outil fournissant une classification cohérente des principales caractéristiques pédagogiques des programmes et instructions et des manuels. Cet outil doit être capable de traduire, dans un langage spécifique commun, les nombreuses particularités nationales dans la définition des objectifs d'apprentissage des mathématiques et des sciences. Les grilles d'analyses des curricula de TIMSS furent conçues pour être de tels outils.

Chaque volet d'un grille d'analyse est organisé de façon hiérarchique utilisant des sous-catégories imbriquées les unes dans les autres dans l'ordre d'une précision croissante. A un niveau donné, le classement des thèmes ne reflète pas un ordre national particulier du contenu (la figure 1, p. 122) donne un aperçu du volet "contenu " des grilles d'analyse des mathématiques et des sciences ${ }^{8}$. Chaque volet d'une grille d'analyse est supposé être encyclopédique pour recouvrir toutes les possibilités à un niveau particulier de précision. La «taille du grain » (grain size) - niveau de précision d'une catégorie - n'est pas

7 D.F. Robitaille, W.H. Schmidt, S. Raizen, C. McKnight, E. Britton et C. Nicol, Curriculum Frameworks for Mathematics and Science, Vancouver, Pacific Educational Press, 1993.

8 On peut trouver la description complète des deux grilles dans Robitaille et al. cité plus haut. 
obligatoirement la même dans toute la grille d'analyse. Certaines sous-catégories sont plus détaillées et communément utilisées que d'autres. Entre les pays participant à TIMSS, il y a de grandes ressemblances mais aussi de grandes variations. Cette "granulation" variable nécessite un soin particulier dans la mise au point de méthodes basées sur les grilles et dans l'interprétation des résultats de leur utilisation.

Dans la grille d'analyse des mathématiques, par exemple, les contenus se répartissent en dix grandes catégories, chacune allant de deux à dix sous-catégories. Certaines sous-catégories sont subdivisées. Le niveau de détail et d'organisation reflète un compromis entre la simplicité (moins de catégories) et la précision (plus de catégories). Les niveaux hiérarchiques de la précision croissante permettent quelque souplesse dans le degré de détail et la généralisation.

Comme nous l'avons déjà dit, les grilles d'analyse permettent une caractérisation complexe des éléments curriculaires. C'est-à-dire que la signature d'un élément curriculaire donné peut être formée de codes provenant de un ou plusieurs aspects de la grille d'analyse. Des signatures complexes révèlent des différences importantes dans la façon dont les curricula sont construits pour atteindre leurs objectifs. Elles signalent les différences dans la façon dont les éléments d'une discipline sont combinés - et les différences sur ce que l'on attend des élèves. Chaque grille d'analyse peut présenter la discipline comme un "chemin » thématique complet, avec un riche ensemble de compétences espérées pour les élèves - comme le recommandent les réformateurs de curricula de beaucoup de pays. Cependant, des signatures simples sont aussi permises, par exemple celles souvent associées aux curricula plus traditionnels et à beaucoup de tests classiques de connaissance.

\section{Mesures longitudinales: suivi d'un thème au long de la scolarité}

Une première étape importante de la caractérisation du curriculum d'un pays est de saisir l'ampleur (breath) de la couverture d'un contenu. C'està-dire, comprendre comment l'enseignement d'un sujet donné est d'abord introduit, comment il est intensifié et comment le thème apparaît dans le curriculum au fil de la scolarité pré-universitaire.

Dans TIMSS, la technique appelée suivi d'un thème au long de la scolarité (topic trace mapping) fournit une "trace " (map) multiniveau de la couverture du contenu ${ }^{9}$. Il a été demandé à des spécialistes du curriculum de chaque

9 Une précédente analyse des méthodologies décrites ici est publiée dans : W.H. Schmidt, "L'analyse de curriculum dans le cadre de la TIMSS : description longitudinale de l'enseignement des disciplines ", Perspectives, revue trimestrielle de l'éducation, 1992, 22 (3), p. 367-376. 
pays, utilisant leur connaissance des instructions et programmes nationaux, d'indiquer pour chacune des catégories les plus fines des grilles d'analyse de mathématiques et de sciences :

- l'âge des élèves auquel le thème est introduit ;

- la tranche d'âge des élèves pendant laquelle l'enseignement du thème est dispensé ;

- l'âge des élèves auquel l'enseignement du thème est intensifié (c'està-dire prend une plus grande importance).

La figure 2 (p. 126) montre des «traces» (tracings ou mappings) de la couverture du curriculum formel de deux thèmes mathématiques représentatifs dans des pays choisis pour illustrer la diversité typique des données. Un «-» indique que des aspects du thème font partie de l'enseignement de mathématiques pour les élèves de cet âge, selon les spécialistes. Un « + » indique que des aspects du thème font l'objet d'un enseignement approfondi - et d'une certaine façon, d'une attention et d'une ampleur curriculaire particulières - pour les élèves de cet âge.

Le thème «congruence et similitude» fait référence aux figures congruentes et semblables - plus spécialement aux triangles, quadrilatères et polygones - et à leurs propriétés. La figure 2 montre, par exemple, que l'enseignement de ce thème est concentré seulement sur deux années d'enseignement en Espagne, pour les élèves de 11 ans et de 12 ans. Au contraire, les spécialistes hongrois indiquent que dix années d'enseignement sont prévues pour ce thème, en commençant avec les élèves de 8 ans, avec une importance plus grande pour ceux de 13 et 16 ans. D'autres modèles émergent, allant des pays, comme l'Argentine, prévoyant seulement une année sur ce thème en second cycle de l'enseignement secondaire (upper secondary), aux pays, comme le Canada et la Hongrie, qui prévoient une couverture du thème presque depuis le début de l'enseignement primaire jusqu'à la fin du second cycle de l'enseignement secondaire. D'autres pays présentent des modèles de couverture "interrompus » ou «multisites " - par exemple Chypre et le Japon. Les modèles montrés dans cette figure sont représentatifs des autres pays et de beaucoup d'autres thèmes.

Le thème "équations et formules" présente une nette différence. Il comprend tout contenu algébrique en relation avec des équations. Les spécialistes de la plupart des pays indiquent que ce thème est enseigné du tout début de l'enseignement primaire jusqu'à la fin de l'enseignement secondaire spécialisé, ce qui est logique pour un thème aussi vaste. En effet, certains pays, comme par exemple l'Irlande et la Nouvelle-Zélande, l'introduisent très tôt.

La «trace » d'un thème est basée sur l'opinion de spécialistes de chaque pays et seulement indirectement sur les instructions et programmes officiels. Cependant la méthode fournit clairement une première étape essentielle de la compréhension du contexte de la couverture des contenus formels pour chaque niveau ciblé par TIMSS pour les tests de connaissance. Pour illustrer la valeur 
Figure 2. Deux " traces» du suivi d'un thème au long de la scolarité

Ces données sont des "traces" typiques d'un échantillon de pays choisis pour représenter la diversité. Les résultats sont représentatifs d'autres pays et d'autres thèmes.

\begin{tabular}{|c|c|c|c|c|c|c|c|c|c|c|c|c|c|}
\hline \multicolumn{14}{|c|}{ Congruence et similitude } \\
\hline \multicolumn{14}{|c|}{ Âges } \\
\hline & 6 & 7 & 8 & 9 & 10 & 11 & 12 & 13 & 14 & 15 & 16 & 17 & 18 \\
\hline Argentine & & & & & & & & & + & & & & \\
\hline Canada & - & - & - & - & - & - & - & - & - & - & - & - & \\
\hline Chypre & & & & & & & + & & - & + & & & \\
\hline Danemark & & & & & & & + & - & - & + & + & - & \\
\hline Hongrie & & & - & - & - & - & - & + & - & - & + & - & \\
\hline Islande & & & & & & & & + & - & - & + & - & \\
\hline Iran & & & & & & + & - & - & + & - & - & & \\
\hline Irlande & & & & & & & & + & + & + & & - & + \\
\hline Japon & & & & & + & + & & + & - & & & & \\
\hline $\begin{array}{l}\text { Nouvelle- } \\
\text { Zélande }\end{array}$ & & & & & & & - & - & - & - & - & & \\
\hline Espagne & & & & & & + & + & & & & & & \\
\hline Tunisie & & & & & & & & + & - & - & - & - & + \\
\hline USA & & & & & & - & - & + & - & + & & & \\
\hline
\end{tabular}

\begin{tabular}{|c|c|c|c|c|c|c|c|c|c|c|c|c|c|c|}
\hline \multicolumn{15}{|c|}{ Équations et formules } \\
\hline \multicolumn{15}{|c|}{ Âges } \\
\hline & 5 & 6 & 7 & 8 & 9 & 10 & 11 & 12 & 13 & 14 & 15 & 16 & 17 & 18 \\
\hline Argentine & & & & & & & & & & & + & + & - & \\
\hline Canada & & & & & - & - & - & - & + & + & + & + & + & - \\
\hline Chypre & & - & - & - & - & - & - & - & + & + & + & + & + & \\
\hline Danemark & & & - & - & - & + & - & - & + & + & + & + & + & - \\
\hline Hongrie & & & & - & - & + & - & - & + & - & - & + & & \\
\hline Islande & & & & & & & & & - & - & + & - & + & - \\
\hline Iran & & & & & & & + & - & - & - & + & & & \\
\hline Irlande & - & - & - & - & - & - & - & - & + & + & + & & + & + \\
\hline Japon & & & & + & + & + & + & + & + & + & + & + & + & \\
\hline $\begin{array}{l}\text { Nouvelle- } \\
\text { Zélande }\end{array}$ & - & - & - & - & - & - & - & - & - & + & + & + & + & \\
\hline Espagne & & & & & & & & - & - & - & - & - & - & \\
\hline Tunisie & & & & & & & & & & - & + & + & + & + \\
\hline USA & & - & - & - & - & - & + & + & + & - & + & + & & \\
\hline
\end{tabular}

de ces données, prenons par exemple les programmes et instructions en Hongrie et en Islande qui indiquent probablement des objectifs différents pour l'enseignement des congruences et similitudes aux élèves de 13 ans. Cette différence provient du fait que le thème est introduit pour la première fois en Islande aux 
élèves de 13 ans alors que les élèves hongrois de 13 ans doivent en avoir commencé l'étude plusieurs années avant. De même, l'absence du thème " équations et formules » pour les élèves de 9 ans en Islande et en Iran - contrairement, par exemple, aux États-Unis et au Danemark où les spécialistes indiquent que l'enseignement commence plus tôt - implique probablement une différence dans la façon de traiter le thème (si toutefois il est enseigné) dans les manuels des élèves en Islande et en Iran.

Les données collectées par la méthode du suivi d'un thème au long de la scolarité permettent une évaluation initiale de la variété et de la succession des objectifs de couverture des contenus dans les pays participant à TIMSS. Bien qu'elle fournisse des informations essentielles, la méthode a de notables inconvénients. Premièrement, elle se base sur l'opinion des spécialistes. Deuxièmement, lors des expérimentations, cette méthode n'a pas fourni d'information utile sur les compétences espérées et les perspectives. Par chance, le suivi des thèmes au long de la scolarité n'est qu'une composante d'un ensemble de méthodes multiples qui se concentrent principalement sur l'analyse détaillée des programmes et instructions officiels et des manuels scolaires.

\section{Méthode d'analyse de documents}

La caractérisation détaillée du curriculum formel est aussi très importante. Une technique appelée analyse de document (document analysis) a été mise au point pour fournir une mesure en profondeur du curriculum formel de certaines populations cibles de l'enseignement primaire et secondaire ${ }^{10}$. Des échantillons nationalement représentatifs des programmes et instructions officiels et des manuels scolaires ont été sélectionnés et des méthodes nouvellement élaborées ont été utilisées pour réunir les données à partir de chaque document.

\section{Éléments de la méthodologie ${ }^{11}$}

L'échantillon de documents était vaste, de plus, avec presque 50 pays impliqués, la diversité linguistique des documents était déconcertante. On a mis au point des méthodes de manière à ne pas traduire de grandes parties des documents. Cependant le succès et l'efficacité de l'analyse de documents ne reposaient pas uniquement sur le fait d'éviter des traductions massives. D'autres obstacles logistiques nécessitaient la garantie que toutes les procédures opéra-

10 Voir la note $n^{\circ} 6$.

11 Ce vaste ensemble de matériaux décrivant tous les aspects du recueil des données, des techniques d'analyse et l'estimation de la fiabilité est disponible en contactant: Dr G.A. Valverde, Associate Director, Survey of Mathematics and Science Opportunities, Michigan State University, 457 Erickson Hall, East Lansing, Michigan 48824-1034, USA. 
Figure 3. Exemple de pages d'un manuel

Ces deux pages sont extraites d'un manuel de mathématiques destiné au niveau le plus élevé de la population 2. Elles représentent une partie d'une unité. L'équipe nationale d'analyse de documents a indiqué le numéro et le type de l'unité et des blocs.

\section{RESOLUCIÓN DE UN SISTEMA DE ECUACIONES POR MIETODOS}

\section{ALGEBRAICOS}

Para resolver un sistema de dos ecuaciones con dos Incógnilitos. ademáśs del métrodo grúfico aue ya

\section{Método de sustitución}

a) Analiza el desarrollo del sigulente problema.
2 se tienen $\$ 500$ en monedas de $\$ 5$ y de $\$ 20$. $\$ 1$ dieron 55 monedas ¿cuántas son de $5 y$
cuántas de 20 ?

Sea $x$ la cantidad de monedas de $\$ 5$ y $m$ la cantidad de monedas de $\$ 20$. T. 6

$5 x$ representa la cantidad de dinero en monedas de $\$ 5$

$20 \mathrm{~m}$ representa la cantldad en monedas de $\$ 20$.

$$
5 x+20 m=500
$$

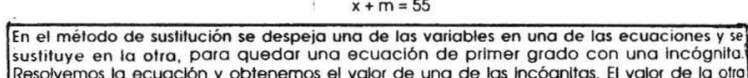
variable lo obtenemos al sustituir el valor conocido en la ecuación donde quedó despejado la otra incógnita.

$$
\begin{aligned}
& 5 x+20 m=500 \ldots \text { (1) } \\
& x+m=55 \ldots \\
& \text { En la ecuación } 2 \text {, despejamos } x \\
& x+m=55 \\
& \begin{aligned}
-m & =\frac{-m}{x}=55-m
\end{aligned} \\
& 275-5 m+20 m=500 \\
& \begin{aligned}
275+15 m & =500 \\
2 / 2 & =275 \\
15 m & =1
\end{aligned} \\
& m=\overline{15}
\end{aligned}
$$$$
5 x+20 m=500
$$$$
5(55-m)+20 m=500
$$

Iñ Temas de Álgebra
Si sabemos que $x=55-m$, sustitulmos $m$ por su valor.

$$
x=55-15
$$$$
[x=40]
$$

Comprobaclón

$$
5 x+20 m=500
$$

$x+m=55$

$5(40)+20(15)=$

$$
40+15=
$$

andenedas de 5 Y 15 monedas de 520

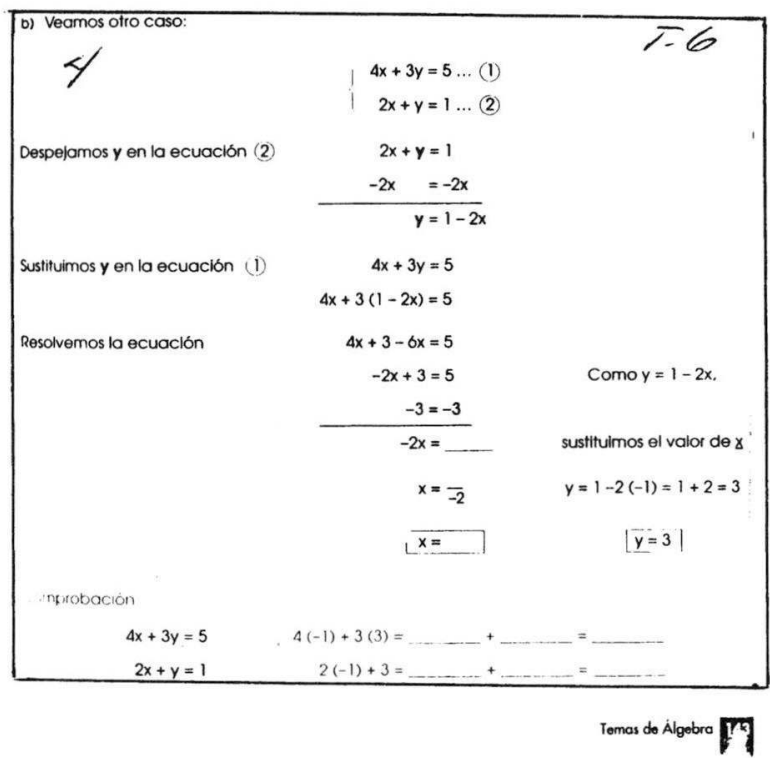


tionnelles y compris le codage, le marquage, le report et l'archivage avaient été totalement précisées, que les codeurs «autochtones» de chaque pays avaient été entraînés à appliquer les procédures (qui comprenaient la production de formulaires de données codées avec des textes en anglais) et que ces données - à la fois codées et écrites en anglais, aussi bien pour les programmes et les manuels étaient saisies dans une banque de données centrale, nettoyées, éditées et vérifiées par le pays les ayant fournies.

\section{Identification d'une "unité »}

La première étape du processus d'analyse de document est la division de chaque document en petites unités (units) sur lesquelles portent des analyses plus fines. Dans les programmes, ces unités sont les plus petites parties fonctionnelles du document - par exemple, il y a les unités d'introduction, celles présentant la politique, celles énonçant les objectifs éducatifs, celles spécifiant les contenus mathématiques ou scientifiques, celles précisant les objectifs d'apprentissage et les "autres unités 12 ».

Dans les manuels, l'unité la plus fondamentale est la leçon. Une leçon est définie comme une partie du texte consacrée à un seul thème principal mathématique ou scientifique et destinée à correspondre à une leçon du professeur en classe sur le thème enseigné pendant une à trois heures de cours. Quelques parties des manuels (par exemple, les révisions) recouvrent le contenu de plusieurs leçons et sont classées comme des pages de leçons multiples. Ces deux types d'unités avec l'introduction, les annexes pédagogiques et les «autres unités " constituent les cinq types d'unités d'un manuel. Chaque équipe nationale collectant les données a identifié et assigné un type à chaque unité du manuel.

\section{Identification des «blocs»}

Diviser les documents en larges unités fonctionnelles simplifie l'analyse mais ne rend pas assez compte de tous les détails de structure nécessaires pour construire une description statistique des documents curriculaires - ou au moins de certaines de leurs caractéristiques clés. Ainsi, les unités sont-elles subdivisées en petits blocs (blocks). La figure 3 (p. 128) montre, sur l'exemple d'un manuel, comment la procédure des blocs est appliquée.

La figure 3 reproduit deux pages d'un manuel de mathématiques pour le niveau le plus haut de la population 2 (élèves de 13 ans). Ce sont les deux premières pages de l'unité $\mathrm{n}^{\circ} 33$, une leçon simple (unité de type 2 - "T2 " ici). L'unité porte sur la résolution de systèmes d'équations par des méthodes algé-

12 Sous le terme « autres unités " sont regroupées les diverses unités, peu nombreuses, n'entrant pas dans les types cités précédemment. (N.D.T.). 
briques, les deux pages présentées traitant de la méthode de substitution. En suivant les règles de la partition standardisée, l'équipe de codage a divisé ces deux pages en blocs, encadrés et numérotés de 1 à 4 .

Chaque unité de tous les programmes et manuels sélectionnés a été divisée de la même façon en segments plus petits (les blocs). En identifiant différents types de blocs d'une part pour les manuels et d'autre part pour les programmes, nous avons pu construire un résumé statistique plus détaillé de chaque document. Pour les programmes, les types de blocs portent sur les politiques, les objectifs, les éléments particuliers de contenu, les suggestions pédagogiques, les exemples liés aux suggestions pédagogiques, les suggestions d'évaluation et autres types de blocs. Pour les manuels, les types de blocs sont les blocs narratifs (explications pédagogiques centrales ou secondaires, liées entre elles ou non), les blocs graphiques liés aux blocs narratifs, les blocs graphiques informatifs non directement liés aux blocs narratifs pédagogiques, les exercices et questions (directement liés ou non au contenu d'une unité - par exemple, les ensembles de révisions insérés dans une unité mais sur un thème différent), les activités proposées, les exercices corrigés, les " autres » blocs du manuel.

La division des unités en blocs et l'identification des types de blocs suivent des règles précises. Ces règles sont exposées dans les rapports techniques de TIMSS. La figure 3 en donne un exemple. Quatre blocs sont indiqués et numérotés sur les deux pages (on peut remarquer que le bloc 3 est à l'intérieur du bloc 4). Chaque type de bloc est indiqué - successivement types 1, 6, 1 et 6 . Le bloc 1 est une courte partie narrative, dont la traduction approximative est " résoudre un système de deux équations à deux inconnues, avec la méthode graphique que l'on vient de voir [dans des pages non présentées ici], on peut utiliser d'autres procédures de nature algébrique». Les titres fournissent d'autres informations - que ce qui suit porte sur "la méthode de substitution " et que le bloc 2 ( « a. analyser la résolution du problème suivant ») et le bloc 4 («b. Voici d'autres exemples») sont des exercices corrigés que l'élève doit étudier. A l'intérieur du premier exercice corrigé (bloc 2), il y a un bloc narratif qui commence par «Avec la méthode de substitution, une des variables d'une équation a été trouvée... ».

La stratégie des premières pages de cette unité est claire. Une constatation d'ordre général est que la méthode algébrique, aussi bien que les méthodes graphiques, peut être utilisée pour résoudre un système d'équations. On trouve ici un exercice corrigé portant sur la méthode de substitution. A l'intérieur de cet exemple, une courte partie narrative résume la stratégie à suivre dans la méthode de substitution. Un deuxième exercice corrigé est présenté. Le premier exemple est un problème contextualisé portant sur des pièces de monnaies, le second est un exercice de mathématiques situé hors de tout contexte. Toutes ces précisions devaient être résumées sous la forme d'éléments permettant de voir 
les points communs aux différents pays et populations. Un de ces éléments saisi par le codage est le type du bloc, indiquant ici que la stratégie de ces deux pages est mise en œuvre au travers de parties narratives et d'exercices corrigés (blocs de types 1 et 6 ).

\section{Codage}

La division en unités et blocs dont les types sont précisés est au centre de la préparation des résumés de documents. Ces données et leur relation avec les pages des documents sont enregistrées. Les contenus, les compétences espérées et les perspectives se rapportant à chaque bloc sont aussi identifiées. Après la division d'un document en unités et blocs, chaque bloc est décrit par des codes de catégories de chaque volet des grilles d'analyse de mathématiques ou de sciences. Ces codes - aussi bien ceux des blocs que des unités - sont les principales informations reportées dans le résumé statistique de chaque document. D'autres informations descriptives sont aussi fournies au travers de questionnaires portant sur le document, l'unité et le bloc.

La figure 4 (p. 132) montre comment une équipe nationale de collecte des données a consigné ces catégories sur des formulaires dont les colonnes correspondent aux blocs à l'intérieur de chaque unité. Cette figure est la reproduction d'un formulaire complet de codage utilisé par les codeurs pour enregistrer les liens entre les pages du document, les numéros des blocs, les types de blocs et les codes de la grille d'analyse des mathématiques. Les quatre premiers blocs de ce formulaires sont ceux des pages de la figure 3. Les quatre premières colonnes correspondent à ces quatre blocs. Les types de blocs sont repérés par des codes numériques ( 1 et 6 ) et liés aux numéros des pages du manuel et des blocs. Chacun des blocs a reçu un code simple de contenu, 1.6.2, qui se réfère à l'algèbre des équations. Les deux blocs narratifs, $\mathrm{n}^{\circ} 1$ et 3 , sont associés au même code, 2.1.3, de compétence espérée, code "par défaut » pour indiquer que ces blocs sont des parties à lire. Les blocs d'exercices corrigés, $n^{\circ} 2$ et 4 , reçoivent le code, 2.2.2., " application de procédures courantes " indiquant que c'est ce que l'on attendrait des élèves s'ils devaient résoudre eux-mêmes les exercices. Un seul code de perspective est utilisé - utilisation des relations entre les mathématiques et la vie de tous les jours pour intéresser les élèves - code qui est attribué à l'exercice corrigé portant sur la monnaie. Typiquement, les codes de perspective ont été rarement utilisés. Dans cet exemple, tous les blocs ont des signatures très simples; seul un bloc du formulaire est associé à plus d'un code pour un aspect particulier de la grille d'analyse.

La complexité des signatures des blocs - c'est-à-dire le nombre de catégories de chaque volet de la grille d'analyse associées à un bloc - est importante. Des signatures complexes révèlent des niveaux plus élevés de complexité de l'intégration de la discipline dans les programmes et les manuels. 
Figure 4. Exemple de formulaire de codage

Ce formulaire rempli contient les blocs des pages de la figure $3 ; y$ sont indiqués les numéros des pages où se situe le bloc, son numéro d'identification, le numéro de son type et les catégories qui lui correspondent dans la grille d'analyse.

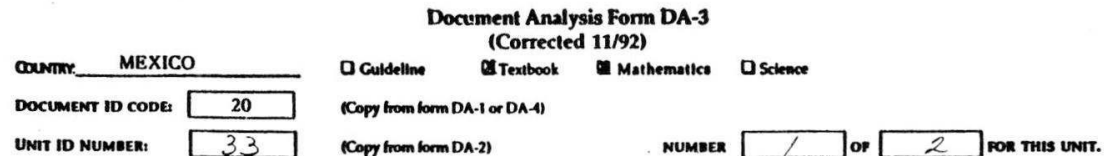

\begin{tabular}{|c|c|c|c|c|c|c|c|c|c|c|}
\hline Prese on & 142 & $|4|-142$ & 142 & 143 & 144 & 144 & $145-147$ & 145 & $147-148$ & 148 \\
\hline Block to & 1 & 2 & 3 & 4 & 5 & 6 & 7 & 8 & 9 & 10 \\
\hline stoct inse & 1 & 6 & 1 & 6 & 6 & 6 & 6 & 1 & 6 & 6 \\
\hline $\begin{array}{c}\text { Contem } \\
\text { costes }\end{array}$ & 1.6 .2 & 1.6 .2 & 1.6 .2 & 1.62 & 1.6 .2 & 1.62 & 1.6 .2 & 1.6 .2 & 1.6 .2 & 1.6 .2 \\
\hline Secondeng & & & & & & & & & & \\
\hline Primang & 2.13 & 2.2 .2 & $2 \cdot 1 \cdot 3$ & 2.2 .2 & 2.2 .2 & 2.3 .3 & $\begin{array}{l}2.3 .1 \\
22.2\end{array}$ & 2.1 .3 & 2.2 .2 & $2.3 \cdot 3$ \\
\hline Secondy & & & & & & & & & & \\
\hline $\begin{array}{l}\text { Pernpocivef } \\
\text { costes (3...) }\end{array}$ & $\phi$ & 3.4 & $\varnothing$ & $\phi$ & $\phi$ & 3.4 & $\phi$ & $\varnothing$ & $\phi$ & 3.4 \\
\hline $\begin{array}{l}\text { ock inpecen } \\
\text { 1. Offk } \\
\text { 2. Obir } \\
\text { 3. Com }\end{array}$ & $\begin{array}{l}\text { des lor Currik } \\
\text { cial Policies } \\
\text { centes } \\
\text { ent elememe }\end{array}$ & $\begin{array}{l}\text { Lum Guildex } \\
\text { 4. Pedagogical } \\
\text { 5. Examples } \\
\text { 6. Assessment } \\
\text { 7. O'ther }\end{array}$ & & $\begin{array}{c}\text { Block ty } \\
1 . \\
2 . \\
3 . \\
4 .\end{array}$ & $\begin{array}{l}\text { pe codes lor Tex } \\
\text { Narrative } \\
\text { Related narrati } \\
\text { Unrelated instn } \\
\text { Relaled graphic }\end{array}$ & $\begin{array}{l}\text { tibook Materials } \\
\text { ve } \\
\text { uctional namativ } \\
c\end{array}$ & $\begin{array}{l}\text { 5. Unor } \\
\text { 6. Enem } \\
\text { 7. Unre } \\
\text { ex }\end{array}$ & 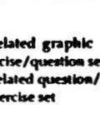 & 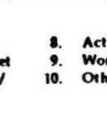 & $\begin{array}{l}\text { Ivily } \\
\text { ried example } \\
\text { ier }\end{array}$ \\
\hline
\end{tabular}

\section{Intégration des mesures}

La spécification complète des objectifs d'apprentissage à un niveau national ou régional nécessiterait une analyse de tous les programmes et instructions, ainsi que celle de tous les manuels disponibles à chaque niveau scolaire et pour chaque série ou filière d'un système éducatif donné. Une telle collecte de données dans une étude internationale n'est ni judicieuse ni réalisable. Il y a tout simplement trop de détails, trop de manuels et trop de niveaux scolaires (et dans certains pays, trop de séries).

Étant donné la situation, on a dû utiliser un processus de mesures soigneusement mis au point. Les deux types de mesures décrits dans cet article représentent deux des composantes les plus importantes du système de mesures mis au point pour TIMSS par SMSO ${ }^{13}$. L'utilisation combinée de ces mesures

13 Des mesures supplémentaires existent, par exemple, pour caractériser l'organisation du curriculum formel des systèmes éducatifs (cours offerts en mathématiques, différenciations des curricula de mathématiques et de sciences dans les différents types d'établissements - tels que les écoles professionnelles, par exemple, etc.) et les prises de décision concernant le curriculum. 
dans les rapports de TIMSS concilie le besoin de profondeur dans la spécification des objectifs d'apprentissage aux niveaux cibles où les données de réussite furent recueillies dans l'étude, avec le besoin d'extension dans la description des objectifs d'apprentissage au long de la scolarité - reconnaissant ainsi la nature cumulative du curriculum.

En liaison avec les autres mesures du curriculum formel et aussi avec la diversité des mesures du curriculum mis en ouvre (y compris les nouvelles mesures des objectifs et pratiques pédagogiques des professeurs) et du curriculum atteint (utilisant les tests de connaissance en mathématiques et en sciences qui furent mis au point à l'aide des analyses préliminaires des données curriculaires internationales), TIMSS est en mesure de beaucoup contribuer à la recherche en éducation et à la prise de décisions politiques dans beaucoup de pays en fournissant des indicateurs qui nous permettront de comprendre les liens entre les objectifs, la réalité des établissements et des classes et la réussite des élèves. 